\title{
New experimental approaches to investigate the fission dynamics
}

\author{
J. Benlliure ${ }^{1, a)}$, J.L. Rodríguez-Sánchez ${ }^{1}$, H. Alvarez-Pol ${ }^{1}$, L. Audouin ${ }^{2}$, Y. Ayyad ${ }^{1}$, \\ G. Bélier ${ }^{3}$, G. Boutoux ${ }^{2}$, E. Casarejos ${ }^{4}$, A. Chatillon ${ }^{3}$, D. Cortina-Gil ${ }^{1}$, T. \\ Gorbinet $^{3}$, A. Heinz ${ }^{5}$, A. Kelić-Heil ${ }^{6}$, B. Laurent ${ }^{3}$, J.-F. Martin ${ }^{3}$, C. Paradela ${ }^{1}$, E. \\ Pellereau $^{3}$, B. Pietras ${ }^{1}$, D. Ramos ${ }^{1}$, C. Rodríguez-Tajes ${ }^{1,7}$, D. M. Rossi ${ }^{6}$, H.

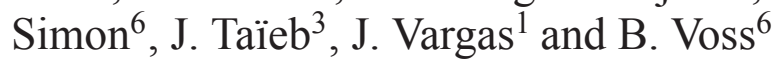 \\ ${ }^{1}$ Universidade de Santiago de Compostela, 15782 Santiago de Compostela, Spain \\ ${ }^{2}$ Institut de Physique Nucléaire d'Orsay, F-91406 Orsay, France \\ ${ }^{3}$ CEA, DAM, DIF, F-91297 Arpajon, France \\ ${ }^{4}$ Universidad de Vigo, E-36200 Vigo, Spain \\ ${ }^{5}$ Chalmers University of Technology, SE-412 96 Gothenburg, Sweden \\ ${ }^{6}$ GSI-Helmholtzzentrum für Schwerionenforschung GmbH, D-64291 Darmstadt, Germany \\ ${ }^{7}$ Grand Accélérateur National d'Ions Lourds, F-14076 Caen Cedex 05, France \\ a)Corresponding author: j.benlliure@usc.es
}

\begin{abstract}
The first ever achieved full identification of both fission fragments, in atomic and mass number, made it possible to define new observables sensitive to the fission dynamics along the fission path up to the scission point. Moreover, proton-induced fission of ${ }^{208} \mathrm{~Pb}$ at high energies offers optimal conditions for the investigation of dissipative, and transient effects, because of the high-excitation energy of the fissioning nuclei, its low angular momentum, and limited shape distortion by the reaction. In this work we show that the charge distribution of the final fission fragments can constrain the ground-to-saddle dynamics while the mass distribution is sensitive to the dynamics until the scission point.
\end{abstract}

\section{INTRODUCTION}

Despite the apparent simplicity of the fission process, and the fact that first models describing this reaction mechanism were proposed soon after its discovery [1] more than 75 years ago, the complete understanding of fission still represents a real challenge for nuclear physicists. This is justified by the complexity of a process where microscopic, and macroscopic degrees of freedom are coupled. Moreover, one needs to understand not only the static properties of fission, governed by the corresponding potential-energy landscape, but also the dynamics.

Fission dynamics can be interpreted as a diffusion process of the collective coordinates along the energy-potential landscape. It was firstly pointed by Kramers [2] that such a diffusion is governed by the coupling between intrinsic, and collective degrees of freedom that one can describe in terms of a dissipative process. A dissipation parameter determines how fast the fission coordinate reaches the saddle point, the so called transient time, and the saddle-toscission time. A time-dependent description of this dissipative process was provided by Grangé and Weidenmüller in the 80 's by using transport calculations based on the Langevin equation [6]

Those ideas were confirmed in the 90's by measuring multiplicities of neutrons [3], light-charged particles [4], and gamma rays [5] emitted until the scission point. It was found that those multiplicities were much larger than the ones predicted by the statistical model. However, the interpretation of those measurements was not conclusive on the magnitude of the dissipation parameter, and its possible dependencies on temperature and deformation[3, 7, 8]. Possible reasons are entrance channel effects, inherent to fusion-fission reactions mostly used in these investigations,

\footnotetext{
Latin American Symposium on Nuclear Physics and Applications AIP Conf. Proc. 1753, 030014-1-030014-8; doi: 10.1063/1.4955355 Published by AIP Publishing. 978-0-7354-1411-2/\$30.00
} 
deficiencies in the model calculations used for the interpretation of the measurements, or the fact that only few observables were used to constrain a non negligible number of parameters in those calculations.

In order to overcome this situation, the use of fragmentation or spallation reactions investigated in inverse kinematics was proposed. The boosting of the fission fragments induced by the inverse kinematics made it possible to fully identify in atomic, and mass number one of the final fission fragments using a high resolving power magnetic spectrometer [9], or identify in atomic number both fragments using large acceptance ionization chambers [10]. Moreover, fragmentation or spallation reactions produce fissioning systems with high excitation energy, moderate angular momentum, and small shape distortion, fulfilling the optimal conditions for the investigation of dissipative effects in fission $[6,11]$.

The fission of stable actinides or pre-actinides, such as ${ }^{238} \mathrm{U}[12,13],{ }^{208} \mathrm{~Pb}[14],{ }^{197} \mathrm{Au}[15],{ }^{181} \mathrm{Ta}[16]$, or even non stable pre-actinides [17], was investigated using this experimental technique. These works shown that the charge distributions of the final fission fragments, and the corresponding partial fission cross sections can be used to constrain dissipative effects from the initial configuration of the fissioning system until the saddle point. Model calculations determined that the value of the reduced dissipation parameter describing those observables in all these reactions was $\beta=4.510^{21} \mathrm{~s}^{-1}$.

In the present work we go a step further, both in the experimental technique, and the investigation of the fission dynamics. We used a new generation experimental setup giving access, for the first time, to the complete identification of both fission fragments in atomic and mass number. This new experimental capabilities made it possible to define new observables sensitive to the fission dynamics up to the scission point that were used to characterize the friction parameter at small and large deformations.

\section{EXPERIMENTAL SETUP AND MEASUREMENTS}

The main challenges of the present research program was to have access to observables requiring the complete identification in atomic and mass number of both fission fragments. This was achieved by using the inverse kinematics technique, and a new generation detection setup SOFIA [18]. An schematic representation of this detection setup is depicted in Fig. 1 where the different detectors and the large acceptance dipole are shown.

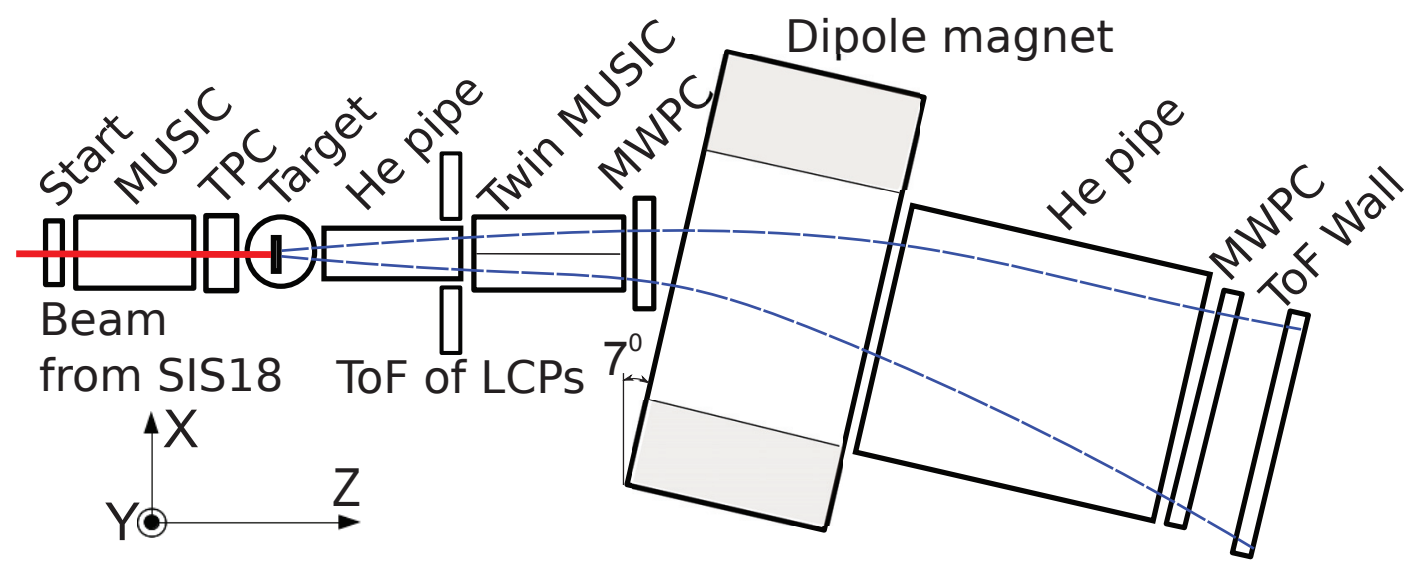

FIGURE 1. Schematic representation of the SOFIA detection setup including all the detectors used for the identification of the fission fragments, the helium pipes to reduce the energy straggling and the large acceptance dipole magnet. See text for a detailed description.

The experimental setup is divided in two parts, one used to characterize the incoming beam ions, and another dedicated to measure the fission fragments. The first part consists of a plastic scintillator detector (start) [26] used to measure the time-of-flight (ToF) of the fragments, a multi-sampling ionization chamber (MUSIC) [27], and a time projection chamber (TPC) [28]. These last two detectors provide the beam identification, and its position on the target, respectively.

The second part consists of a double multi-sampling ionization chamber (Twin MUSIC) [29], two multi-wire proportional counters (MWPCs) [31, 30], a large acceptance dipole magnet (ALADIN), and a ToF Wall [26]. The 
Twin MUSIC chamber, filled with P25 (74.5\% of Argon, $25 \%$ of $\mathrm{CH}_{4}$, and $0.5 \%$ of $\mathrm{CO}_{2}$ ) gas, has a central vertical cathode that divides its volume (60 cm long, $20 \mathrm{~cm}$ high and $20 \mathrm{~cm}$ wide) into two active parts, segmented in ten anodes each. These anodes provide ten independent energy-loss, and drift-time measurements which allow to obtain the atomic numbers with a resolution below 0.43 charge units full width at half maximum (FWHM), and the angles on the plane $X-Z$ with a resolution below $0.6 \mathrm{mrad}$ (FWHM). MWPCs situated in front and behind the dipole magnet, provide the horizontal $(X)$, and vertical $(Y)$ positions of the fission fragments. The MWPC situated in front of the dipole magnet $(20 \mathrm{~cm}$ high and $20 \mathrm{~cm}$ wide) provides the $X$ and $Y$ positions with a resolution around $200 \mu \mathrm{m}$ and $1.5 \mathrm{~mm}$ (FWHM), respectively, while the MWPC situated behind the dipole magnet $(60 \mathrm{~cm}$ high and $90 \mathrm{~cm}$ wide) provides those positions with a resolution around $300 \mu \mathrm{m}$ and $2 \mathrm{~mm}$ (FWHM), respectively. Both MWPCs were filled with a mixture of Argon (80\%) and $\mathrm{CO}_{2}(20 \%)$. Finally, a ToF Wall $(60 \mathrm{~cm}$ high and $90 \mathrm{~cm}$ wide) made of 28 plastic scintillators $(3.2 \mathrm{~cm}$ wide, $60 \mathrm{~cm}$ long and $0.5 \mathrm{~cm}$ thick) allows to measure the ToF of the fission fragments with respect to the start signal provided by the plastic scintillator, located at the entrance of the experimental setup, with a resolution around $40 \mathrm{ps}$ (FWHM) [26]. The ALADIN magnet was set to a magnetic field of 1.6 T, and its gap (200 $\mathrm{cm}$ long, $50 \mathrm{~cm}$ high and $100 \mathrm{~cm}$ wide) was filled with helium gas at atmospheric pressure.

In addition, two pipes, also filled with helium gas at atmospheric pressure, were mounted in front of the Twin MUSIC chamber, and behind the dipole magnet ALADIN. The pipes were sealed by windows made of aluminizedmylar foils with a thickness of $35 \mu \mathrm{m}$ and $50 \mu \mathrm{m}$, respectively. In all the cases, the helium gas was employed to reduce the energy and angular straggling of the fission fragments.

Finally, light-charged particles emitted in coincidence with fission fragments were identified using a ToF wall detector (ToF of LCPs), placed in front the Twin MUSIC chamber. This detector consists of two detection planes of segmented plastic-scintillators (50 cm long, $6 \mathrm{~cm}$ wide and $1 \mathrm{~cm}$ thick), one with six horizontal paddles, and another with six vertical paddles which leave a square hole $\left(12.5 \times 12.5 \mathrm{~cm}^{2}\right)$ in the center for the transmission of the fission fragments.

Full identification of the fission fragments was made using a ray-tracing method coupled to GEANT4 simulations to reconstruct their mass numbers. In the simulation, we took into account the composition, dimensions, and positions of the detectors, as well as the magnetic field, and helium gas inside the dipole magnet ALADIN. Outside of the magnet we assumed that fission fragments follow straight-line trajectories.

The reconstruction of the trajectories was done using the measured atomic numbers, positions, and angles of the fission fragments before the dipole magnet ALADIN, as well as the positions on the target obtained with the TPC, and tracking capabilities of the setup as an input for the simulation. Then, different trajectories were simulated for each fission fragment covering the expected range of $B \rho$, between 6-10 Tm. The simulated trajectories were used to parametrize the flight path length, and $B \rho$ of each fission fragment, as a function of the position of the MWPC situated behind the dipole magnet. Then, this parametrization together with the experimental position recorded by the MWPC, were used to obtain the experimental flight path length, and $B \rho$ of each fission fragment. Finally, the measured $\mathrm{ToF}$, and the reconstructed flight path length were used to deduce the velocity of the fragments with a resolution of $\Delta v / v \sim 0.11 \%$ (FWHM).

Consequently, the mass number $(A)$ was obtained for each fission fragment from its magnetic rigidity, velocity and atomic number, according to the equation:

$$
A=\frac{e Z}{u} \frac{B \rho}{\beta \gamma c}
$$

where $Z$ is the atomic number provided by the Twin MUSIC detector, $B$ is the magnetic field inside the magnet, $\rho$ is the radius of the trajectory, $\mathrm{u}$ is the atomic mass unit, e is the electron charge, $\gamma=1 / \sqrt{1-v^{2} / c^{2}}, v$ is the velocity of the ion, and $\mathrm{c}$ is the velocity of light.

Using this procedure, the mass number of each fission fragment was obtained. The absolute calibration in mass number was deduced using previous measurements [32] as reference. The final average resolution achieved in mass number was $\triangle A / A \sim 0.63 \%$ (FWHM).

\section{GROUND-TO-SADDLE DYNAMICS}

The almost complete kinematics measurements performed in this work give us the possibility to define observables sensitive to the dynamics of fission for different deformation values. Small deformations can be investigated by obtaining information from the dynamical evolution from the initial deformation of the fissioning nuclei up to the saddle 

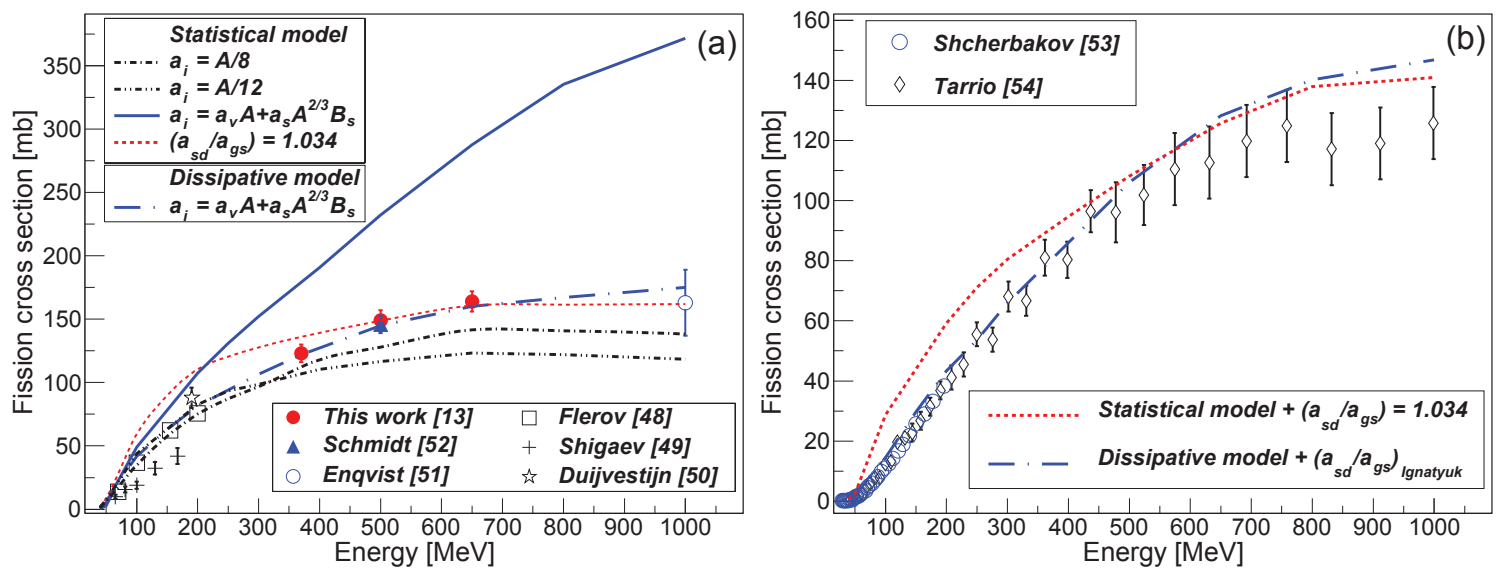

FIGURE 2. (a) Comparison of the total fission cross sections for the reaction ${ }^{208} \mathrm{~Pb}+p$ as a function of the bombarding energy with different model calculations (lines). The data are taken from Refs. [35, 36, 37, 34, 33]. (b) As (a) but for the the reaction ${ }^{\text {nat }} \mathrm{Pb}$ $+n$. The data are taken from Refs. $[38,39]$.

point. The most widely used observable characterizing the fission process at the saddle deformation is the fission probability. In our case we determined the fission cross sections normalizing the number of fission events to the beam flux and target thickness. Moreover, we correct the measured fission yields by the detection efficiency and possible multiple reactions in the target. A detailed description of this procedure can be found in Ref. [19].

In the left panel of Fig. 1 we display as solid points the total fission cross sections measured in this work at $370 \mathrm{~A}$, $500 \mathrm{~A}$ and $650 \mathrm{~A} \mathrm{MeV}$. In the same figure we also show other measurements in inverse kinematics at $1000 \mathrm{~A} \mathrm{MeV} \mathrm{[33]}$ and $500 \mathrm{~A} \mathrm{MeV} \mathrm{[34],} \mathrm{and} \mathrm{in} \mathrm{direct} \mathrm{kinematics} \mathrm{below} 200 \mathrm{MeV}$ [35, 36, 37]. As shown in the figure, the measured cross section at $500 \mathrm{~A} \mathrm{MeV}$ is in perfect agreement with the previous measurement by Schmidt and collaborators [34], and our energy dependence also seems to be in good agreement with other measurements at higher and lower energies.

In order to investigate the dynamics of the fission process up to the saddle point, we use state-of-the-art calculations performed with the intra-nuclear cascade code INCL [40] coupled to the deexcitation code ABLA07 [23]. In intra-nuclear cascade codes, the collision of a high-energy projectile within a target nucleus initiates a succession of binary nucleon-nucleon collisions that may lead to the emission of pre-equilibrium nucleons. In INCL and ISABEL, nucleons are described as point-like particles, and as a continuous medium perturbed by the collisions induced by cascade particles, respectively. Nucleons in INCL are endowed within a realistic phase-space density (Woods-Saxon in configuration space, hard Fermi sphere in momentum space), while in ISABEL, both projectile and particle follow a folded Yukawa density distribution. In INCL, the excitation energy and the angular momentum induced by the intra-nuclear cascade are computed according to particle-hole excitations. The cascade stops self-consistently when the prefragment is assumed to be thermalized. In contrast, in ISABEL the cascade stops when the energy is below a specific cutoff energy. In both codes, the remaining excitation energy is distributed among all the nucleons.

The ABLA07 code describes the deexcitation of a thermalized compound nucleus by fission or by the emission of light charged particles, $\gamma$-rays, neutrons, and intermediate-mass fragments (IMF) following Weisskopfs model [41]. The fission probability is computed according to a time-dependent fission width following the analytical description of the solutions of the corresponding Fokker-Plank equation proposed in Refs. [24, 11, 25]. The code also allows to evaluate the fission width according to the transition-state model of Bohr and Wheeler [1] or the time-independent formulation of Kramers [2]. Important parameters in the code are the level density parameters that are calculated according to the parametrization proposed by Ignatyuk [42], and angular momentum dependent fission barriers which are taken from Sierks finite-range liquid drop model [43]. For the dynamical description of the fission width based on the Fokker-Planck equation another important ingredient is the friction parameter governing the energy transfer rate between intrinsic and collective degrees of freedom.

The results of these calculations are depicted by the different lines shown in Fig. 2. In these calculations we investigate the role of two different ingredients in the model, level densities and the dissipation parameter. As can be seen, different combinations of the values adopted for the level density parameter and the friction parameter could provide a reasonable description of the measured cross sections. Although the best description of the data corresponds 

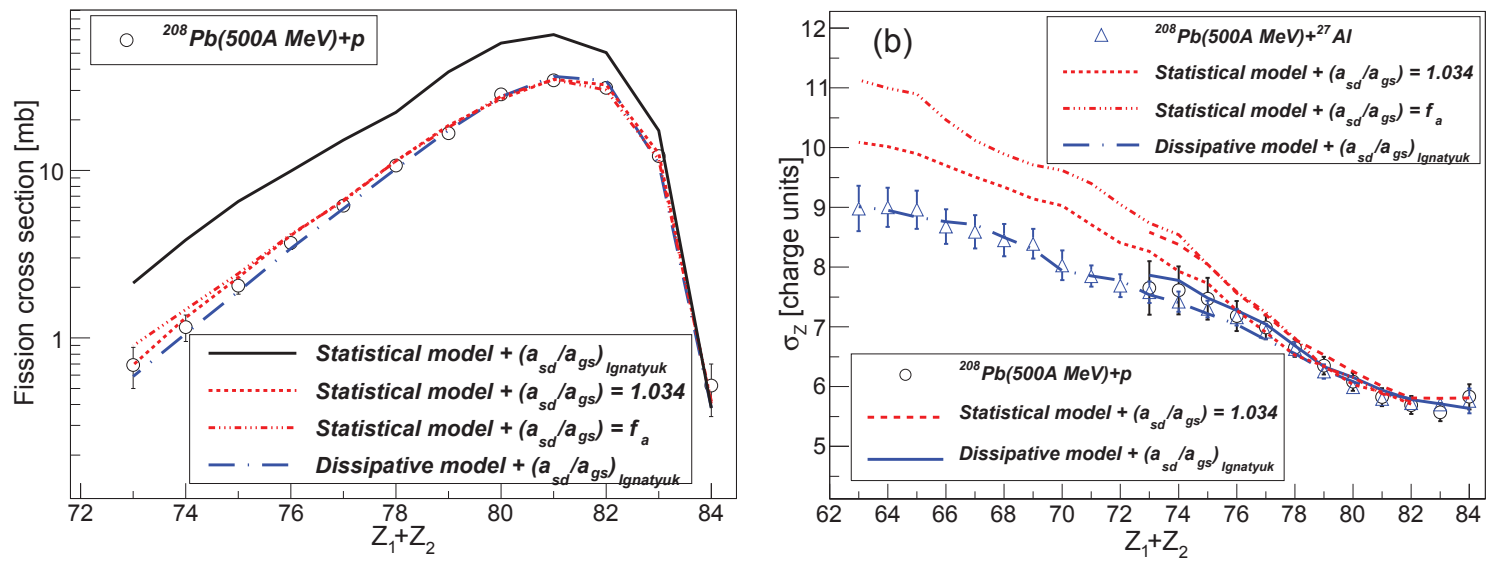

FIGURE 3. (a) Fission cross section of the reaction ${ }^{208} \mathrm{~Pb}+p$ at $500 \mathrm{~A} \mathrm{MeV}$ as a function of the atomic number of the fissioning nuclei (open circles). The lines represent different model calculations. (b) Width of the atomic-number distribution of the final fission fragments measured in spallation and fragmentation reactions of ${ }^{208} \mathrm{~Pb}$ as a function of the atomic number of the fissioning nuclei for different targets (protons (points) and ${ }^{27} \mathrm{Al}$ (open triangles) and projectile energies. The lines represent different model calculations.

to a calculation using a dynamical description of the fission width with a value of the friction parameter around $\beta$ $=4.510^{21} \mathrm{~s}^{-1}$, and a deformation dependent description of the level density parameter according to the prescription proposed by Ignyatyuk [42].

Similar results are obtained in the right panel of Fig. 2 where total fission cross sections from neutron-induced fission reactions on ${ }^{n a t} \mathrm{~Pb}$ are shown, and in the left panel of Fig. 3 where we depict partial fission cross sections according to the sum of the atomic numbers of the final fission fragments. Considering that proton emission is negligible after the saddle point because of the large neutron excess of the fission fragments, the final charge of the final fission fragments should be representative of the charge of the fissioning nuclei at saddle. Therefore, most of the protons should be lost during the intra-nuclear cascade phase and the difference in charge between the initial nucleus and the final fission fragments should be correlated with the excitation energy gained by the nucleus in the collision. Even in this later case the results are not conclusive. Our conclusion was that the use of a single observable can not unambiguously constrain fission models. This could explain the large discrepancies in the need of dissipative and transient effects in fission, and even in the magnitude of the friction parameter.

In order to overcome this situation we proposed the use of additional observables sensitive to the fission dynamics up to the saddle point. In particular we used the correlation between the width of the charge distribution of the fission fragments and its total charge. According to a statistical interpretation, the fluctuation in charge of the fissioning nuclei should be governed by the curvature of the potential describing the fission process in charge asymmetry and the temperature. Because the charge of the final fragments should not be very different to the charge of the fissioning system at saddle, the width of the charge distribution of the fission fragments should provide information on the temperature of the fissioning system at saddle. This hypothesis is supported by the fact that this width does not depend on the entrance channel of the reaction as shown in the right panel of Fig. 3. Moreover, the calculations shown in this figure indicate the inadequacy of statistical models for describing the fission process and clearly constrain the description of the level density parameter.

Once we have fixed the description of the level density parameter with the width of the charge distribution of the fission fragments, we can investigate the magnitude of the dissipation parameter using the partial fission cross sections as a function of the charge of the fissioning nucleus as shown in the left panel of Fig. 4. The calculations in this figure clearly show the sensitivity of this observable to the dissipation parameter at small deformation obtaining the best description for a value $\beta_{g s}=4.510^{-21} \mathrm{~s}^{-1}$. 

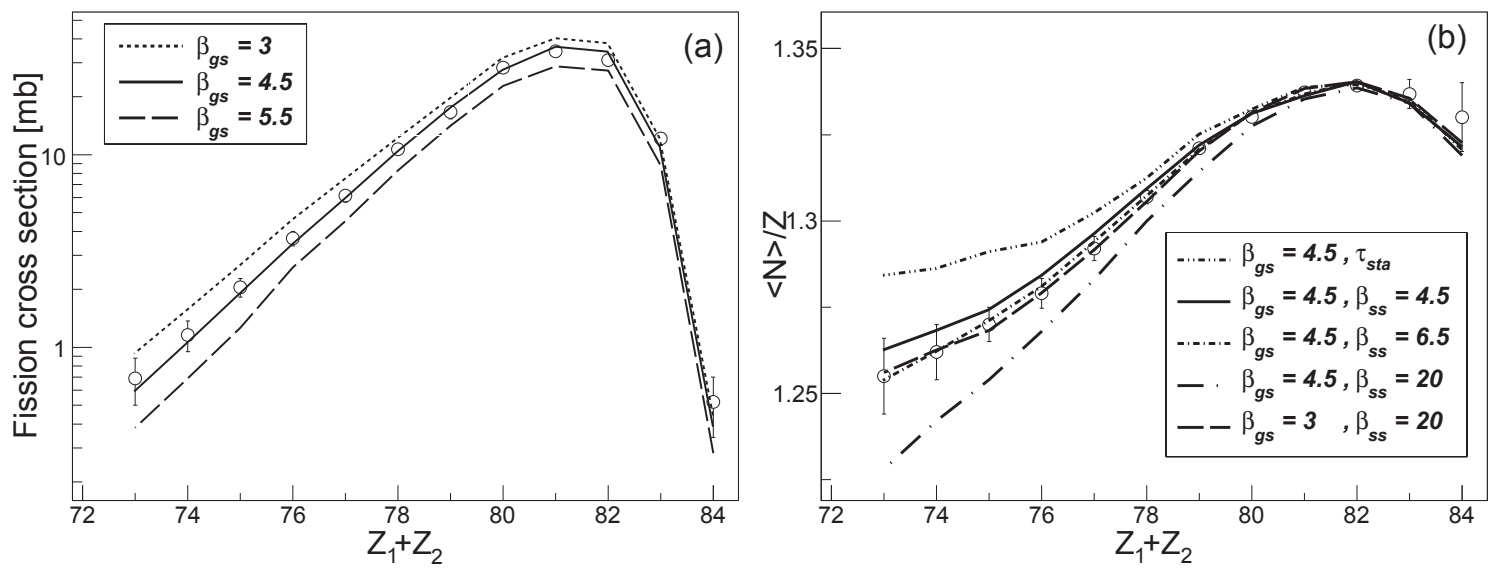

FIGURE 4. (a) Fission cross sections of the final fission fragments as a function of the atomic number of the fissioning nuclei. (b) Average neutron excess of the final fission fragments as a function of the atomic number of the fissioning nuclei. The lines represent model calculations for different values of the dissipation parameters from ground-to-saddle (gs) and saddle-to-scission (ss) in units of $10^{21} \mathrm{~s}^{-1}$.

\section{SADDLE-TO-SCISSION DYNAMICS}

The description of the saddle-to-scission dynamics has been mostly addressed in the past by using the multiplicities of emitted pre-scission neutrons. Following this idea, we proposed to use as alternative the neutron excess of the final fission fragments. Although the final neutron excess of the fragments also depends on the post-scission neutron emission, the saddle-to-scission emission should clearly influence this observable. The final sensitivity will depend on the accuracy of the model description of pre-saddle and post-scission neutron emission. On the other hand, this is an observable that we can determine with high accuracy using our experimental setup.

In the right panel in Fig. 4 we display the neutron excess of the final fission fragments as a function of their total charge, together with several model calculations. To include the fission dynamics beyond the saddle point in our model calculations, we have used the pioneering model proposed by Hofmann and Nix [48], who found an analytical solution for the average saddle-to-scission time obtained with the dynamical formulation of Kramers [2]. This dynamical time can be compared with the statistical evaporation time obtained from the sum of the particle decay widths to determine the number of particles emitted between the saddle and scission points. If the saddle-to-scission time of the fissioning system is longer than the statistical evaporation time, the fissioning system could emit additional particles during that phase, mainly neutrons and $\gamma$-rays, increasing the pre-scission particle multiplicities. The intra-nuclear cascade and the evaporation models used in these calculations have been previously validated describing the residual nuclei production in spallation reactions on non fissile targets, such as ${ }^{56} \mathrm{Fe}[44]$ and ${ }^{136} \mathrm{Xe}[45,46,47]$.

The calculations shown in Fig. 4.b clearly indicate again the inadequacy of using an statistical model for describing the saddle-to-scission time. Concerning the calculations based on transport equations, the benchmark also shows the sensitivity of the neutron excess of the final fragments to the value of the saddle-to-scission dissipation parameter. These calculations also show that different combinations of the ground-to-saddle and saddle-to-scission dissipation parameter can reproduce the data. Therefore, one needs an accurate determination of the ground-to-saddle dissipation parameter, using an independent observable, in order to conclude about the saddle-to-scission dynamics. In our case, once we have fixed the ground-to-saddle dissipation parameter, using the partial fission cross sections $\left(\beta_{g s}=4.510^{-21}\right.$ $\mathrm{s}^{-1}$ ), we obtain the best description of the neutron excess of the final fragments using a saddle-to-scission dissipation parameter $\beta_{s s}=6.510^{-21} \mathrm{~s}^{-1}$.

Several conclusions are reached from the present work. First, we demonstrate that one needs several independent observables to characterize the different parameters in the model calculations. In particular, one needs an accurate characterization of the ground-to-saddle dynamics before any realistic determination of the saddle-to-scission dissipation parameter can be obtained. Moreover, we obtain almost the same value for the dissipation parameter at small and large deformations. Therefore, we do not observe any significant dependence of the dissipation parameter with deformation. We also describe all the observables used in this work with the same value of the dissipation parameter over a 
broad range of excitation energy. This result also indicates that this parameter neither present a strong dependence on temperature.

\section{CONCLUSIONS}

The SOFIA experiment at GSI represents a real break through in the investigation of nuclear fission. 75 years after the discovery of fission, both fission fragments were fully identified in atomic and mass number for the first time, taking advantage of the inverse kinematics technique and a state-of-the-art detection setup. This experiment makes then possible almost complete kinematics measurements. In this work we have presented the first experiment using the SOFIA setup to investigate the fission dynamics at high excitation energy, using proton induced fission reactions on ${ }^{208} \mathrm{~Pb}$. Those are the conditions expected to better show dynamical and transient effects in fission.

In this experiment we were capable to define several independent observables constraining the dynamics of the fission process along the fission path. In particular, we used partial fission cross sections according the the total charge of the final fission fragments to determine the dissipation parameter needed to describe the fission dynamics from ground-to-saddle deformation $\beta_{g s}=4.510^{-21} \mathrm{~s}^{-1}$.

We also proposed a new observable to investigate the fission dynamics from the saddle to the scission point, the neutron excess of the final fission fragments. It was also shown that post-saddle dynamics can only be characterized after an accurate determination of the properties of the fissioning system at saddle. The neutron excess of the fission fragments was shown to be an observable sensitive to the dissipation parameter at large deformation that was better describe using a value of $\beta_{s s}=6.510^{-21} \mathrm{~s}^{-1}$.

The similitude between the values of the dissipation parameters obtained at small and large deformation allowed us to conclude that this parameter does not present any strong dependence on deformation, contrary to previous results. Moreover, the description of the measured observables over a broad range in excitation energy also seems to indicate that the dissipation parameter does not depend on temperature.

\section{ACKNOWLEDGMENTS}

The authors are grateful to the GSI accelerator staff for providing an intense and stable beam of ${ }^{208} \mathrm{~Pb}$. We also acknowledge J.-M. Gheller and S. Leray for providing the liquid hydrogen target and N. Kurz and A. Prochazka for their technical support during the experiment. This work was partially supported by the European Commission under Projects No. ANDES-FP7-249671 and No. CHANDA-FP7-605203, the Spanish Ministry of Research and Innovation under Projects No. FPA2010-22174-C02 and No. Consolider-CPAN-CSD2007-00042 and the Regional Government of Galicia under the program "Grupos de Referencia Competitiva 2013-011".

\section{REFERENCES}

[1] N. Bohr and J. A. Wheeler, Phys. Rev. 56, 426 (1939).

[2] H. A. Kramers, Physica 7, 284 (1940).

[3] D. Hilscher and H. Rossner, Ann. Phys. Fr. (Paris) 17, 471 (1992).

[4] M. Thoennessen and G. F. Bertsch, Phys. Rev. Lett. 71, 4303 (1993).

[5] J. P. Lestone, Phys. Rev. Lett. 70, 2245 (1993).

[6] P. Grangé, Li Jun-Qing, and H. A. Weidenmüller, Phys. Rev. C 27,

[7] D. J. Hofman, B. B. Back, and P. Paul, Phys. Rev. C 51, 2597 (1995).

[8] P. Fröbrich et al., Nucl. Phys. A 556, 281 (1993).

[9] M. Bernas et al., Phys. Lett. B 415, 111 (1997).

[10] K.-H. Schmidt et al., Nucl. Phys. A 665, 221 (2000).

[11] B. Jurado, C. Schmitt, K.-H. Schmidt, J. Benlliure and A. R. Junghans, Nucl. Phys. A 757, 329 (2005).

[12] B. Jurado et al., Phys. Rev. Lett. 93, 072501 (2004).

[13] J. Benlliure, E. Casarejos, J. Pereira and K.H. Schmidt, Phys. Rev. C 74, 014609 (2006).

[14] Y. Ayyad et al., Phys. Rev. C 89, 054610 (2014).

[15] J. Benlliure et al. Nucl. Phys. A 700, 469 (2002).

[16] Y. Ayyad et al., Phys. Rev. C 91, 034601 (2015).

[17] C. Schmitt et al., Phys. Rev. Lett. 99, 042701 (2007). 
[18] J.F. Martin et al., Eur. Phys. J. A 51, 174 (2015).

[19] J. L. Rodríguez-Sánchez et al., Phys. Rev. C 90, 064606 (2014).

[20] J. L. Rodríguez-Sánchez et al., Phys. Rev. C 91, 064616 (2015).

[21] J. L. Rodríguez-Sánchez et al Phys. Rev. C 92, 044612 (2015).

[22] J. L. Rodríguez-Sánchez et al submitted to Phys. Lett. B

[23] A. Kelić, M. V. Ricciardi, and K.-H. Schmidt, Proceedings of Joint ICTP-IAEA Advanced Workshop on Model Codes for Spallation Reactions, ICTP Trieste, Italy, 48 February 2008, edited by D. Filges, S. Leray, Y. Yariv, A. Mengoni, A. Stanculescu, and G. Mank (IAEA INDC(NDS)-530, Vienna, 2008), pp. 181-221.

[24] B. Jurado, K.-H. Schmidt, and J. Benlliure, Phys. Lett. B 553, 186 (2003).

[25] B. Jurado, C. Schmitt, K.-H. Schmidt, J. Benlliure, and A. R. Junghans, Nucl. Phys. A 747, 14 (2005).

[26] A. Ebran et al., Nucl. Instrum. Methods Phys. Res., Sect. A 728, 40 (2013).

[27] M. Pfüzner et al., Nucl. Instrum. Methods Phys. Res., Sect. B 86, 213 (1994).

[28] R. Janik et al., Nucl. Instrum. Methods Phys. Res., Sect. A 640, 54 (2011).

[29] B. Voss, Proceedings of the Nuclear Science Symposium and Medical Imaging Conference (NSS/MIC-IEEE, Valencia, Spain, 2011)

[30] C. Finck et al., J. Phys.: Conf. Ser. 50, 397 (2006).

[31] E. Pellereau et al., EPJ Web Conf. 62, 06005 (2013).

[32] B. Fernández-Domínguez et al., Nucl. Phys. A 747, 227 (2005).

[33] T. Enqvist et al., Nucl. Phys. A. 686, 481 (2001).

[34] K.-H. Schmidt et al., Phys. Rev. C 87, 034601 (2013).

[35] G. N. Flerov et al., Sov. J. At. Energy Vol. 33, p.979 (1972).

[36] O. E. Shigaev et al., Khlopin Radiev. Inst., Leningrad Reports, No. 17 (1973).

[37] M. C. Duijvestijn et al., Phys. Rev. C 59, 776 (1999).

[38] O. A. Shcherbakov et al., J. Nucl. Sci. Technol. 39, 230 (2002).

[39] D. Tarrio et al., Phys. Rev. C 83, 044620 (2011).

[40] A. Boudard, J. Cugnon, J.-C. David, S. Leray, and D. Mancusi, Phys. Rev. C 87, 014606 (2013).

[41] V. Weisskopf, Phys. Rev. 52, 295 (1937).

[42] A. V. Ignatyuk et al., Sov. J. Nucl. Phys. 21, 612 (1975).

[43] A. J. Sierk, Phys. Rev. C 33, 2039 (1986).

[44] C. Villagrasa-Canton et al., Phys. Rev. C 75, 044603 (2007).

[45] P. Napolitani et al., Phys. Rev. C 76, 064609 (2007).

[46] J. Alcantara-Nuñez et al., Phys. Rev. C 92, 024607 (2015).

[47] L. Giot et al., Nucl. Phys. A 899, 116 (2013).

[48] H. Hofmann and J. R. Nix, Phys. Lett. B 122, 117 (1983). 